\title{
Changing trends and serotype distribution of Shigella species in Beijing from 1994 to 2010
}

\author{
Yuanli Mao', Enbo Cui ', Chunmei Bao ${ }^{1}$, Zhenhong Liu', Suming Chen', Juling Zhang ${ }^{1}$, Huan Wang ${ }^{1}$, \\ Chenglong Zhang ${ }^{1}$, Jing Zou ${ }^{2}$, John D Klena ${ }^{3}$, Baoli Zhu ${ }^{2}$, Fen Qu ${ }^{1^{*}}$ and Zhiyun Wang ${ }^{2^{*}}$
}

\begin{abstract}
Shigella species are a common cause of acute diarrheal disease in China. In this study, we characterized the changing trends and serotype distribution of Shigella species in Beijing from 1994 to 2010. A total of 5999 Shigella strains were isolated and serotyped from the 302nd Hospital in Beijing. The annual number of Shigella isolates reached a peak ( $n=1192 ; 19.84 \%)$ in 1996 and then decreased annually, reaching the lowest point $(n=24 ; 0.41 \%)$ in 2010. S. flexneri 2a and S. sonnei were the most frequently isolated Shigella, with their respective isolates making up $53.3 \%$ and $27.6 \%$ of the total. Isolates of $S$. flexneri $4 c$, $4 a$, and $x$ made up $3 \%$ respectively of the total isolates. Significant decreases in percentage of $\mathrm{S}$. flexneri over time were observed. S. sonnei surpassed S. flexneri $2 \mathrm{a}$ as the predominant serotype in 2000. Most isolates were recovered from July to September; $13.6 \%$ of the isolates were recovered from children aged 0 to 5 years, and 16\% were recovered from those aged 21 to 25 years. S. flexneri $2 a$ and 5 were recovered mostly from males $(33.41 \%, p<0.001$; and $0.46 \%, p<0.001 \%$; respectively), whereas $S$. flexneri $2 \mathrm{~b}$ and 6 , and S. sonnei were most often isolated from females. Continuous monitoring of Shigella showed that all 4 species and 27 serotypes were present in Beijing, China, during the study period. The emergence of S. sonnei and the overall decreasing isolation rate of Shigella in Beijing can potentially aid in the development of vaccine and control strategies for shigellosis in the city.
\end{abstract}

Keywords: Shigellosis, Shigella, Serotype, Beijing hospital

\section{Introduction}

Shigella species are a common cause of acute diarrheal disease worldwide, with an estimated 167 million cases per year and resulting in approximately 1.1 million deaths; $97.6 \%$ of the cases occur in developing countries [1]. According to the Chinese National Infectious Disease Internet Reporting System, the annual incidence of shigellosis in China made it rank in the top three of the most notable infectious diseases for four consecutive years (2005 to 2008), with close to 500000 cases of shigellosis per year (http://www.moh.gov.cn); this number is now widely believed to be underestimated [2].

Shigellosis is caused by four species, S. dysenteriae, S. flexneri, S. boydii, and S. sonnei. Shigella species can

\footnotetext{
*Correspondence: qf302@163.com; zhiyun_wang@hotmail.com ${ }^{1}$ Clinical Diagnostic Center, 302nd Hospital of the People's Liberation Army, Beijing 100039, PR China

${ }^{2}$ Institute of Microbiology, Chinese Academy of Sciences, Beijing 100101, PR China

Full list of author information is available at the end of the article
}

be identified by serotyping with group-specific antigens; serotyping is based on structural differences within the O-antigen repeating unit of lipopolysaccharide [3]. A total of 47 serotypes of Shigella have been recognized, including 15 for S. flexneri, 13 for S. dysenteriae, 18 for S. boydii, and a single one for S. sonnei [4]. The distribution of species and serotypes of Shigella is heterogeneous over time and place [5].

The World Health Organization has made the development of a safe and effective vaccine against $S$. flexneri $[1,6-8]$, but the vaccine effectiveness depends on the distribution patterns of local species and serotypes, because only type-specific immunity has been demonstrated in humans [9-12] and moreover cross-serotype protection is controversial $[11,13]$.

According to a previous multicenter study of Shigella diarrhea in six Asian countries, S. flexneri is the most common species in Bangladesh, China, Pakistan, Indonesia, and Vietnam; whereas S. sonnei is predominant in industrialized countries [14]. Two recent reports have indicated

\section{Biomed Central}

(c) 2013 Mao et al.; licensee BioMed Central Ltd. This is an Open Access article distributed under the terms of the Creative Commons Attribution License (http://creativecommons.org/licenses/by/2.0), which permits unrestricted use, distribution, and reproduction in any medium, provided the original work is properly cited. 
that S. flexneri 2a is the most frequently isolated Shigella organism in China $[15,16]$. However, these reports may not be generalizable for the whole China; the time period of these studies is short, and surveillance is performed only in less-developed areas of China.

Little is known about the distribution of Shigella serotypes in Beijing, the political, educational, cultural, and economic center of China with a population of over 30 million. The present study describes the trends in Shigella species and their serotypes isolated from patients with diarrhea in a national infectious disease hospital in Beijing, China, from 1994 to 2010.

\section{Materials and methods}

\section{Study sites and settings}

The location was a clinical diagnostic center at the 302nd Hospital of the People's Liberation Army in Beijing, China. The 302nd Hospital is the largest infectious disease teaching hospital in Beijing, China, with 1300 beds and receiving more than 36400 patients annually. From January 1994 to December 2010, fresh stool specimens were collected from patients with diarrhea and clinically suspected dysentery. The specimens were submitted to the microbiology laboratory of the 302nd Hospital. All experimental research have been performed with the approval of ethics committee of 302nd Hospital of the People's Liberation Army, with reference number 2004013D.

\section{Bacterial isolation}

Samples were cultured for Shigella by streaking diarrheal stools directly onto Salmonella-Shigella agar (Tian Tan Biologic Technology Company, Beijing, China) and incubating for $24 \mathrm{~h}$ at $37^{\circ} \mathrm{C}$. Shigella-like colonies were selected and subcultured on Kligler iron agar (Qingdao Hope Biol-Technology Co., Ltd., Shandong, China). Except for S. flexneri 6 and S. boydii 14, Shigella spp. produce an alkaline slant and an acid butt but do not produce gas or $\mathrm{H}_{2} \mathrm{~S}$. As a species, Shigella organisms are characteristically nonmotile and lack the enzyme lysine decarboxylase.

\section{Serotyping}

Serologic identification was performed by slide agglutination with polyvalent somatic $(\mathrm{O})$ antigen grouping sera, followed by testing with available monovalent antisera for specific identification of serotypes according to the manufacturer's instructions (Denka Seiken, Japan). Only one Shigella isolate per patient per diarrheal episode was included in the analysis.

\section{Statistical analysis}

Statistical comparisons were performed using the CHISS software (version 2001, Yuan YiTang Sci-Tech Co., Ltd., Beijing, China). Categorical data were expressed as percentages and calculated using a chi-square test, and $p \leq 0.05$ was considered statistically significant.

\section{Results}

From 1994 to 2010, a total of 5999 Shigella isolates were collected from 372 inpatients and 5627 outpatients with diarrhea. All patients acquired diarrheal infection in the community, most likely through the ingestion of contaminated food and water. Cases for which the diarrheal infection was acquired through travel or via sexual contact were excluded from the analysis. Among the 5999 Shigella isolates, 12 were of an unknown subgroup (i.e., either not reported, not further typed, or untypeable); a final total of 5987 isolates were further analyzed in this study.

\section{Subgroup trends}

A statistically significant decreasing trend in S. flexneri and an increasing trend in S. sonnei were observed by chi-square analysis $(p<0.01)$ (Figure 1). The trends in Shigella spp. isolated from Beijing between 1994 and 2010 are shown in Figure 2. The recording of annual Shigella isolation began in 1994, and the maximum number of isolates was reported in $1996(\mathrm{n}=1194)$. The annual total number of isolated Shigella organisms had been decreasing since then, reaching a low point in 2008 $(\mathrm{n}=22)$. This trend may be related to the strict hygiene inspection and adequate sanitation during the 2008 Olympic season [17]. Four peaks were observed during the 17-year collection period. Peak 1 appeared in 1996, with subsequent peaks in 1998 (peak 2, $\mathrm{n}=602$ ), 2002 (peak 3, $\mathrm{n}=398$ ), and 2004 (peak $4, \mathrm{n}=251$ ). A sudden decrease in Shigella isolation was observed more in 2003 than in 2002 and 2004; one possible explanation is that resources were redirected to identify severe acute respiratory syndrome cases in China in 2003, thereby limiting bacterial diarrheal isolation. It should be noted that as the numbers of observed cases of shigellosis were decreasing, China's per capita gross domestic product (GDP) was increasing (Figure 3).

\section{Subgroups}

The distribution of typeable Shigella during the study period was S. flexneri, 71.7\% ( $\mathrm{n}=4295)$; S. sonnei, $27.3 \%$ $(\mathrm{n}=1639) ;$ S. dysenteriae, $0.55 \%(\mathrm{n}=33)$; and $S$. boydii, $0.33 \%(\mathrm{n}=20)$. The distribution of Shigella species changed over the 17-year observation period (Figure 4). Between 1994 and 2005, Shigella isolation rates were largely driven by $S$. flexneri, reaching peak numbers in 1996 when $90 \%$ of all isolated Shigella were S. flexneri. In 2006, S. sonnei became the dominant subgroup. In 2009 , the lowest percentage of isolated S. flexneri (6\%) was recorded. The apparent isolation rates of $S$. boydii and $S$. dysenteriae increased during this period, e.g., $S$. 


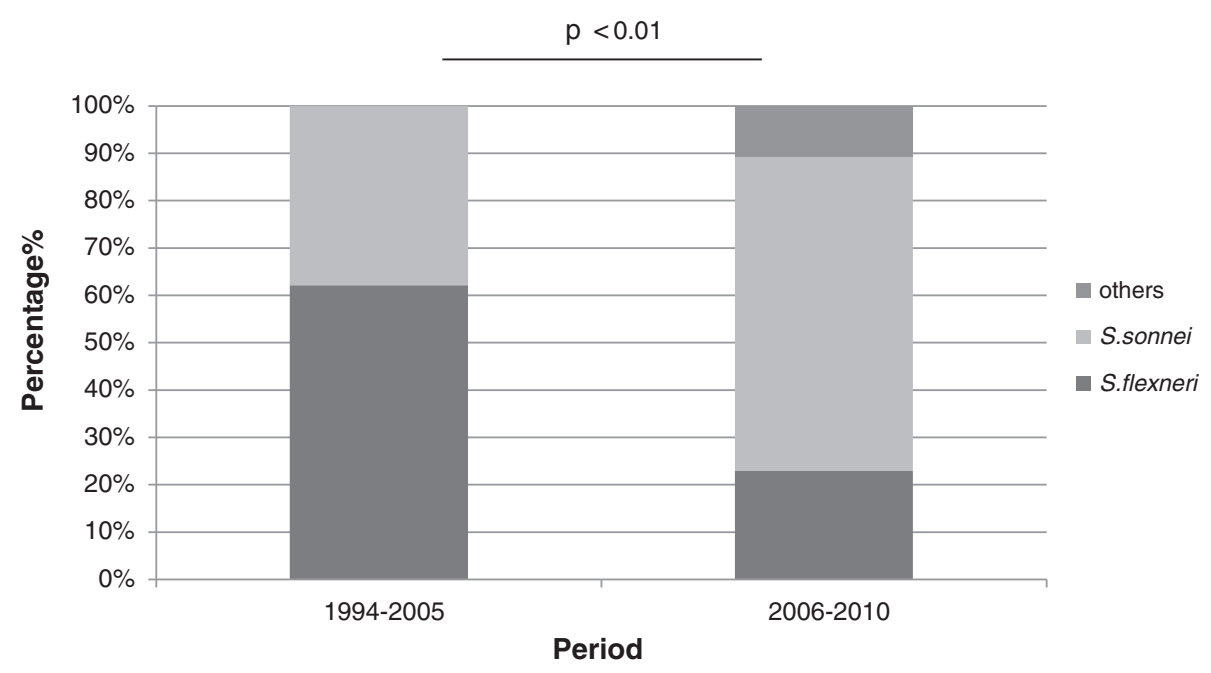

Figure 1 The distribution of Shigella species in Beijing from 1994 to 2010.

boydii isolation rates increased from $0 \%$ to $4.2 \%(\mathrm{n}=5)$, $3.8 \%(\mathrm{n}=3), 4.5 \%(\mathrm{n}=1), 8.3 \%(\mathrm{n}=3)$, and $7.6 \%(\mathrm{n}=2)$ in 2006, 2007, 2008, 2009, and 2010, respectively. However, the absolute numbers of $S$. boydii and $S$. dysenteriae did not change during this period, remaining between 0 and 5 per year. This result suggests that although uncommon, sources of $S$. dysenteriae and S. boydii remain in the Beijing area.

\section{Serotypes of S. flexneri and S. sonnei}

As revealed by 17 years' worth of data, 27/47 (57.4\%) Shigella serotypes were identified; these serotypes included $S$. boydii serotypes $1,2,5,15$, and $17 ; S$. dysenteriae serotypes $1,2,3,4,5,7$, and $8 ; S$. flexneri serotypes 1a, 1b, 2a, 2b, 3a, 3b, 4a, 4b, 4c, 5, 6, x, and y; and $S$. sonnei. Given the small numbers of $S$. boydii and $S$. dysenteriae ( $\mathrm{n}=53$ combined), only isolates of $S$. flexneri and S. sonnei were further analyzed. Surveillance data indicated large differences in the serotypes of $S$. flexneri isolated between 1994 and 2010, in addition to the change in dominant species in 2006 (Table 1). S. flexneri $2 \mathrm{a}$ and S. sonnei were observed in every year of the study, and S. sonnei surpassed S. flexneri 2a as the predominant single Shigella type in 2000. The number and proportion of S. flexneri 2 a isolates decreased from $42.6 \%(n=262)$ in 1994 to $13.6 \%(n=3)$ in 2010, whereas the proportion of $S$. sonnei increased from $36.6 \% \quad(n=225)$ to $82.3 \% \quad(n=20)$ during the entire period of data collection. S. flexneri 2 a ranked the first among the S. flexneri subtypes except in 2003, 2004, and 2005. S. flexneri 4c, 4a, and S. flexneri x were seldom isolated, accounting for only about $3 \%(\mathrm{n}=180)$ of the 5934.S. flexneri and S. sonnei isolates. However, S. flexneri 4c was the most frequently isolated $S$. flexneri

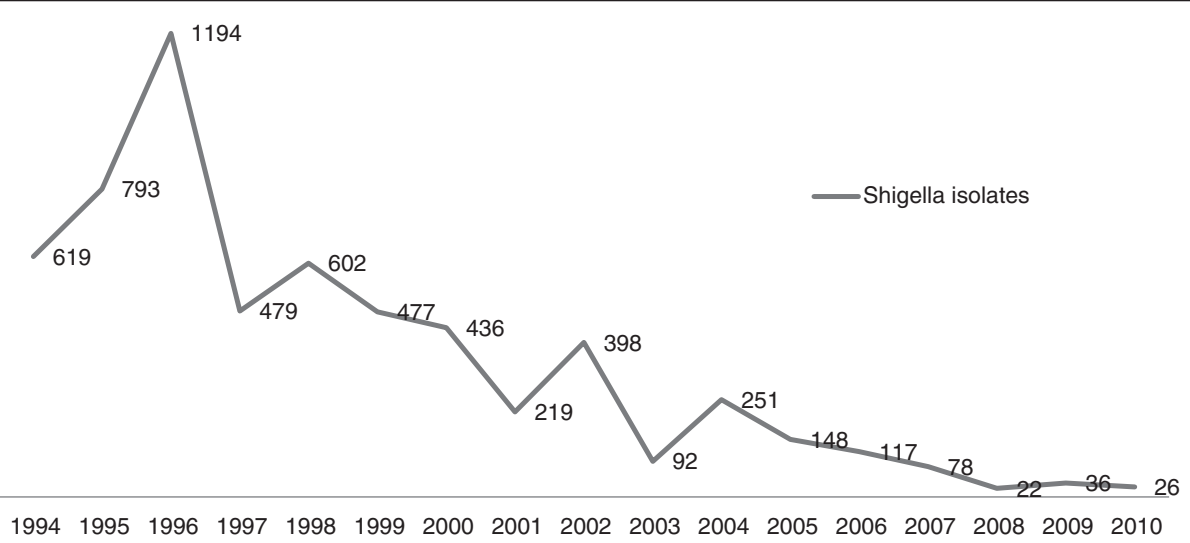

Figure 2 Trends over time for Shigella spp. isolated from Beijing between 1994 and 2010. 


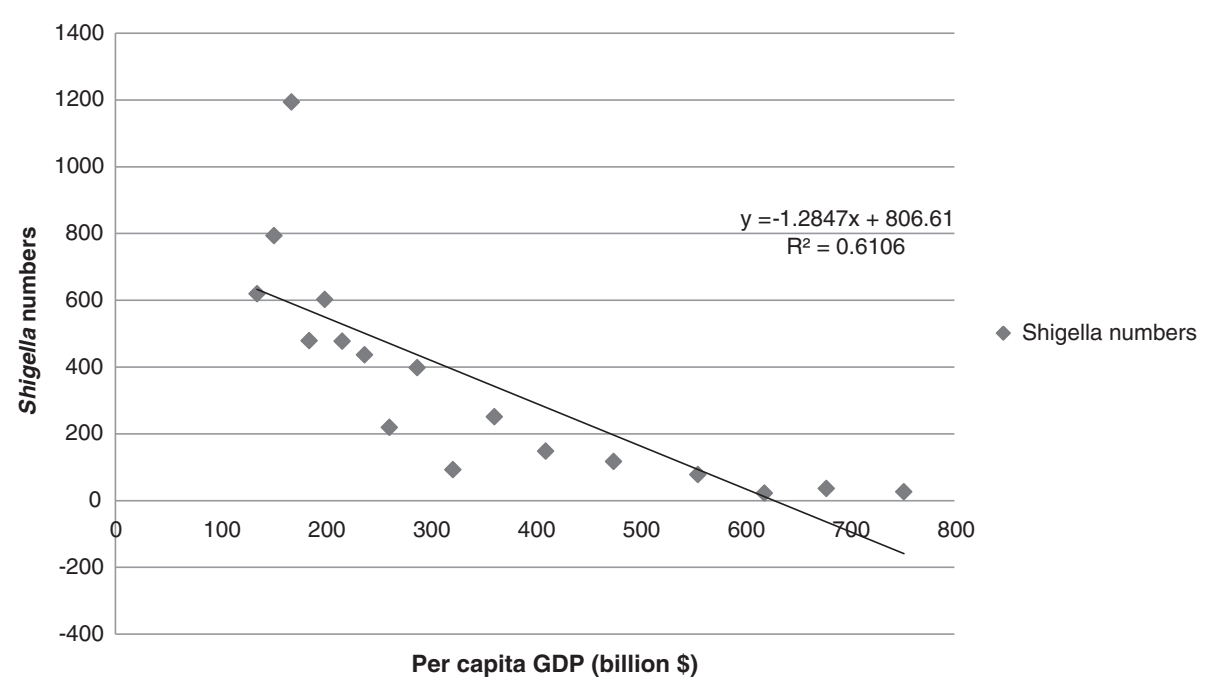

Figure 3 Number of isolates by per capita GDP (adjusted for purchasing power parity) of China from 1994 to 2010.

serotype in $2004(26.4 \%, \mathrm{n}=65)$ and $2005(20.3 \%, \mathrm{n}=$ 30). S. flexneri 4c and S. sonnei were isolated in equal numbers in 2004.

\section{Seasonality}

Shigella isolates were recovered routinely throughout the study but were frequently recovered in the summer months (June to September; $\mathrm{t}=7.83, p<0.001$; Table 2). Isolation of Shigella almost always peaked in July and August; 2003 and 2008 were exceptional years during which a September peak was observed. As indicated in the Beijing weather information, the temperature in September 2003 reached $33.7^{\circ} \mathrm{C}$, the highest on record for the past 42 years (Provided by China Meteorological Data Sharing Service System, http://cdc.cma.gov.cn/home. do,). In September 2008, the amount of rainfall was $98.1 \mathrm{~mm}$, which was twice the amount of rainfall during the same month in 2007 (Provided by China Meteorological Data Sharing Service System, http://cdc.cma.gov. $\mathrm{cn} /$ home.do). Both factors may have contributed to the late seasonal peaks.

\section{Age distribution}

Epidemiological information was available for all 5999 patients. Patient age ranged from 3 months to 90 years. The age distribution for the 5934 cases of S. flexneri and

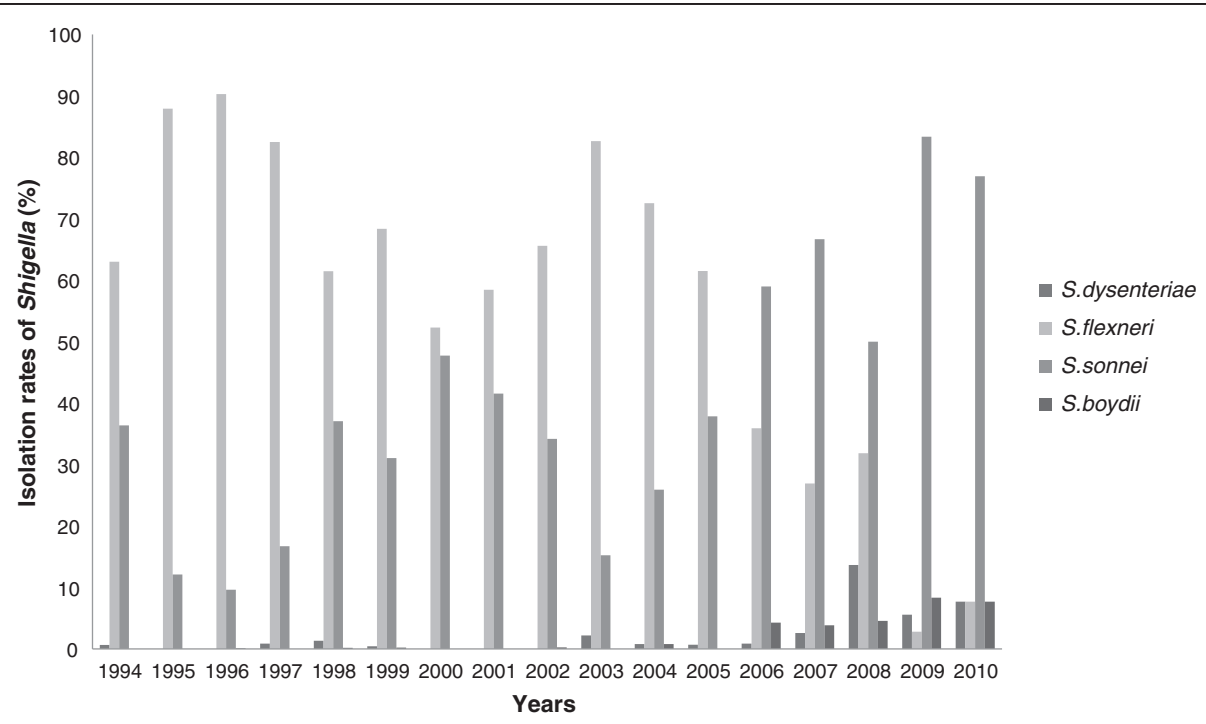

Figure 4 Isolation rates of Shigella spp. by subgroup in Beijing from 1994 to 2010. 
Table 1 Distribution of S. flexneri and S. sonnei serotypes in Beijing between 1994 and 2010

\begin{tabular}{|c|c|c|c|c|c|c|c|c|c|c|c|c|c|c|c|c|}
\hline \multirow{3}{*}{$\begin{array}{c}\begin{array}{c}\text { Surveillance } \\
\text { time }\end{array} \\
1994\end{array}$} & \multicolumn{16}{|c|}{ No. (\%) of isolates } \\
\hline & \multicolumn{2}{|c|}{ Total } & \multicolumn{2}{|c|}{$\begin{array}{l}\text { S. flexneri } \\
\text { serotype 1a }\end{array}$} & \multicolumn{2}{|c|}{$\begin{array}{l}\text { S. flexneri } \\
\text { serotype } 1 \mathrm{~b}\end{array}$} & \multicolumn{2}{|c|}{$\begin{array}{c}\text { S. flexneri } \\
\text { serotype } 2 a\end{array}$} & \multicolumn{2}{|c|}{$\begin{array}{c}\text { S. flexneri } \\
\text { serotype } 2 b\end{array}$} & \multicolumn{2}{|c|}{$\begin{array}{c}\text { S. flexneri } \\
\text { serotype 3a }\end{array}$} & \multicolumn{2}{|c|}{$\begin{array}{c}\text { S. flexneri } \\
\text { serotype } 3 b\end{array}$} & \multicolumn{2}{|c|}{$\begin{array}{c}\text { S. flexneri } \\
\text { serotype 4a }\end{array}$} \\
\hline & 615 & $(10.36)$ & 113 & $(18.37)$ & 0 & $(0.00)$ & 262 & $(42.60)$ & 3 & $(0.49)$ & 0 & $(0.00)$ & 0 & $(0.00)$ & 3 & $(0.49)$ \\
\hline 1995 & 793 & (13.36) & 4 & $(0.50)$ & 1 & $(0.13)$ & 632 & (79.69) & 0 & $(0.00)$ & 2 & $(0.25)$ & 0 & $(0.00)$ & 14 & $(1.77)$ \\
\hline 1996 & 1192 & $(20.09)$ & 5 & $(0.42)$ & 1 & $(0.08)$ & 984 & $(82.55)$ & 0 & $(0.00)$ & 6 & $(0.50)$ & 0 & $(0.00)$ & 23 & $(1.93)$ \\
\hline 1997 & 474 & (7.99) & 2 & $(0.42)$ & 0 & $(0.00)$ & 365 & (77.02) & 0 & $(0.00)$ & 1 & $(0.21)$ & 0 & $(0.00)$ & 4 & $(0.84)$ \\
\hline 1998 & 600 & $(10.11)$ & 1 & $(0.17)$ & 2 & $(0.33$ & 340 & $(56.67)$ & 0 & $(0.00)$ & 0 & $(0.00)$ & 0 & $(0.00)$ & 0 & $(0.00)$ \\
\hline 1999 & 475 & $(8.00)$ & 6 & (1.26) & 3 & $(0.63)$ & 192 & $(40.42)$ & 3 & $(0.63)$ & 11 & $(2.32)$ & 2 & $(0.42)$ & 4 & $(0.84)$ \\
\hline 2000 & 433 & $(7.30)$ & 7 & $(1.62)$ & 1 & $(0.23)$ & 132 & (30.48) & 19 & $(4.39)$ & 7 & $(1.62)$ & 0 & $(0.00)$ & 0 & $(0.00)$ \\
\hline 2001 & 214 & (3.61) & 1 & $(0.47)$ & 1 & $(0.47)$ & 81 & $(37.85)$ & 6 & (2.80) & 0 & $(0.00)$ & 1 & $(0.47)$ & 11 & (5.14) \\
\hline 2002 & 397 & (6.69) & 8 & (2.02) & 0 & $(0.00)$ & 116 & (29.14) & 57 & (14.37) & 0 & $(0.00)$ & 0 & $(0.00)$ & 13 & (3.28) \\
\hline 2003 & 88 & (1.48) & 4 & $(4.55)$ & 2 & $(2.27)$ & 13 & $(14.77)$ & 15 & (17.05) & 0 & $(0.00)$ & 0 & $(0.00)$ & 12 & (13.64) \\
\hline 2004 & 247 & $(4.16)$ & 4 & $(1.62)$ & 1 & $(0.41)$ & 7 & (2.68) & 16 & (6.49) & 3 & $(1.22)$ & 0 & $(0.00)$ & 58 & (23.52) \\
\hline 2005 & 148 & $(2.49)$ & 3 & (2.03) & 0 & $(0.00)$ & 9 & (5.96) & 11 & $(7.44)$ & 0 & $(0.00)$ & 0 & $(0.00)$ & 25 & (16.91) \\
\hline 2006 & 112 & $(1.89)$ & 7 & (6.25) & 1 & $(0.89)$ & 11 & (9.83) & 6 & (5.36) & 0 & $(0.00)$ & 0 & $(0.00)$ & 5 & (4.46) \\
\hline 2007 & 74 & $(1.25)$ & 5 & (6.77) & 0 & $(0.00)$ & 10 & $(13.40)$ & 1 & $(1.35)$ & 0 & $(0.00)$ & 0 & $(0.00)$ & 1 & (1.35) \\
\hline 2008 & 17 & $(0.29)$ & 1 & (5.81) & 0 & $(0.00)$ & 2 & $(12.80)$ & 1 & $(5.81)$ & 0 & $(0.00)$ & 0 & $(0.00)$ & 0 & $(0.00)$ \\
\hline 2009 & 31 & $(0.52)$ & 0 & $(0.00)$ & 0 & $(0.00)$ & 1 & (3.54) & 0 & $(0.00)$ & 0 & $(0.00)$ & 0 & $(0.00)$ & 0 & $(0.00)$ \\
\hline 2010 & 24 & $(0.40)$ & 0 & $(0.00)$ & 1 & $(4.11)$ & 3 & (13.59) & 0 & $(0.00)$ & 0 & $(0.00)$ & 0 & $(0.00)$ & 0 & $(0.00)$ \\
\hline Total & 5,934 & (100) & 171 & (2.88) & 14 & $(0.24)$ & 3,160 & $(53.25)$ & 138 & (2.33) & 30 & $(0.51)$ & 3 & $(0.05)$ & 173 & (2.92) \\
\hline
\end{tabular}


Table 1 Distribution of S. flexneri and S. sonnei serotypes in Beijing between 1994 and 2010 (Continued)

\begin{tabular}{|c|c|c|c|c|c|c|c|c|c|c|c|c|c|c|}
\hline \multirow{3}{*}{$\begin{array}{c}\text { Surveillance } \\
\text { time }\end{array}$} & \multicolumn{14}{|c|}{ No. (\%) of isolates } \\
\hline & \multicolumn{2}{|c|}{$\begin{array}{c}\text { S. flexneri } \\
\text { serotype } 4 b\end{array}$} & \multicolumn{2}{|c|}{$\begin{array}{c}\text { S. flexneri } \\
\text { serotype 4c }\end{array}$} & \multicolumn{2}{|c|}{$\begin{array}{c}\text { S. flexneri } \\
\text { serotype } 5\end{array}$} & \multicolumn{2}{|c|}{$\begin{array}{l}\text { S. flexneri } \\
\text { serotype } 6\end{array}$} & \multicolumn{2}{|c|}{$\begin{array}{l}\text { S. flexneri } \\
\text { serotype } \mathrm{x}\end{array}$} & \multicolumn{2}{|c|}{$\begin{array}{l}\text { S. flexneri } \\
\text { serotype y }\end{array}$} & \multicolumn{2}{|c|}{ S. sonnei } \\
\hline & 3 & $(0.49)$ & 2 & (0.33) & 1 & $(0.16)$ & 2 & $(0.33)$ & 0 & $(0.00)$ & 1 & $(0.16)$ & 225 & (36.59) \\
\hline 1995 & 2 & $(0.25)$ & 10 & $(1.26)$ & 0 & $(0.00)$ & 8 & $(1.01)$ & 5 & $(0.63)$ & 20 & (2.52) & 95 & (11.98) \\
\hline 1996 & 2 & $(0.17)$ & 28 & (2.35) & 1 & $(0.08)$ & 3 & $(0.25)$ & 4 & $(0.34)$ & 21 & $(1.76)$ & 114 & (9.56) \\
\hline 1997 & 1 & $(0.21)$ & 6 & $(1.26)$ & 1 & $(0.21)$ & 0 & $(0.00)$ & 6 & $(1.26)$ & 9 & $(1.90)$ & 79 & (16.65) \\
\hline 1998 & 3 & $(0.50)$ & 4 & $(0.67)$ & 0 & $(0.00)$ & 3 & $(0.50)$ & 8 & $(1.33)$ & 11 & (1.83) & 228 & $(38.00)$ \\
\hline 1999 & 2 & $(0.42)$ & 2 & $(0.42)$ & 2 & $(0.42)$ & 24 & (5.05) & 54 & $(11.37)$ & 23 & $(4.84)$ & 147 & (30.95) \\
\hline 2000 & 2 & $(0.46)$ & 3 & $(0.69)$ & 23 & (5.31) & 8 & (1.85) & 16 & (3.70) & 7 & $(1.62)$ & 208 & (48.04) \\
\hline 2001 & 1 & $(0.47)$ & 15 & $(7.01)$ & 0 & $(0.00)$ & 1 & $(0.47)$ & 4 & (1.87) & 0 & $(0.00)$ & 92 & (42.99) \\
\hline 2002 & 3 & $(0.76)$ & 11 & (2.77) & 1 & $(0.25)$ & 1 & $(0.25)$ & 43 & (10.84) & 8 & (2.02) & 136 & (34.30) \\
\hline 2003 & 7 & (7.95) & 9 & $(10.23)$ & 0 & $(0.00)$ & 0 & $(0.00)$ & 9 & $(10.23)$ & 4 & $(4.55)$ & 13 & (14.77) \\
\hline 2004 & 8 & (3.24) & 65 & $(26.36)$ & 1 & $(0.41)$ & 0 & $(0.00)$ & 17 & (6.89) & 2 & $(0.81)$ & 65 & (26.36) \\
\hline 2005 & 3 & (2.03) & 30 & (20.30) & 0 & $(0.00)$ & 3 & (2.03) & 7 & (4.74) & 1 & (0.68) & 56 & (37.89) \\
\hline 2006 & 7 & (6.25) & 0 & $(0.00)$ & 0 & $(0.00)$ & 0 & $(0.00)$ & 4 & (3.57) & 2 & (1.79) & 69 & (61.60) \\
\hline 2007 & 2 & (2.71) & 1 & (1.35) & 0 & $(0.00)$ & 0 & $(0.00)$ & 1 & (1.35) & 1 & (1.35) & 52 & (70.36) \\
\hline 2008 & 1 & (5.81) & 2 & (11.63) & 0 & $(0.00)$ & 0 & $(0.00)$ & 0 & $(0.00)$ & 0 & $(0.00)$ & 10 & (58.14) \\
\hline 2009 & 0 & $(0.00)$ & 0 & $(0.00)$ & 0 & $(0.00)$ & 0 & $(0.00)$ & 0 & $(0.00)$ & 0 & $(0.00)$ & 30 & (96.46) \\
\hline 2010 & 0 & $(0.00)$ & 0 & $(0.00)$ & 0 & $(0.00)$ & 0 & $(0.00)$ & 0 & $(0.00)$ & 0 & $(0.00)$ & 20 & (82.30) \\
\hline Total & 47 & (0.79) & 188 & (3.17) & 30 & $(0.51)$ & 53 & (0.89) & 178 & (3.00) & 110 & $(1.85)$ & 1,639 & (27.62) \\
\hline
\end{tabular}


Table 2 Distribution of Shigella spp. by Months in Beijing from 1994 to 2010

\begin{tabular}{|c|c|c|c|c|c|c|c|c|c|c|c|c|c|c|c|c|c|c|c|c|c|c|c|c|c|c|}
\hline \multirow{3}{*}{$\begin{array}{c}\begin{array}{c}\text { Surveillance } \\
\text { time }\end{array} \\
1994\end{array}$} & \multicolumn{26}{|c|}{ No. (\%) of isolates } \\
\hline & \multicolumn{2}{|c|}{ Total } & \multicolumn{2}{|c|}{ Jan } & \multicolumn{2}{|c|}{ Feb } & \multicolumn{2}{|c|}{ Mar } & \multicolumn{2}{|c|}{ Apr } & \multicolumn{2}{|c|}{ May } & \multicolumn{2}{|c|}{ Jun } & \multicolumn{2}{|c|}{ Jul } & \multicolumn{2}{|c|}{ Aug } & \multicolumn{2}{|c|}{ Sep } & \multicolumn{2}{|c|}{ Oct } & \multicolumn{2}{|c|}{ Nov } & \multicolumn{2}{|c|}{ Dec } \\
\hline & 620 & $(10.34)$ & 5 & $(0.81)$ & 3 & $(0.48)$ & 3 & $(0.48)$ & 2 & $(0.32)$ & 12 & $(1.94)$ & 56 & $(9.03)$ & 165 & $(26.61)$ & 178 & $(28.71)$ & 122 & (19.68) & 42 & $(6.77)$ & 23 & (3.71) & 9 & $(1.45)$ \\
\hline 1995 & 793 & $(13.22)$ & 10 & $(1.26)$ & 3 & $(0.38)$ & 11 & (1.39) & 16 & (2.02) & 49 & (6.18) & 132 & $(16.65)$ & 239 & $(30.14)$ & 166 & (20.93) & 76 & (9.58) & 46 & (5.80) & 38 & $(4.79)$ & 7 & $(0.88)$ \\
\hline 1996 & 1196 & (19.94) & 25 & (2.09) & 12 & $(1.00)$ & 9 & $(0.75)$ & 5 & $(0.42)$ & 45 & (3.76) & 125 & $(10.45)$ & 376 & (31.44) & 317 & $(26.51)$ & 185 & $(15.47)$ & 61 & (5.10) & 16 & (1.34) & 20 & $(1.67)$ \\
\hline 1997 & 481 & (8.02) & 15 & (3.12) & 5 & $(1.04)$ & 11 & (2.29) & 19 & (3.95) & 55 & (11.43) & 76 & (15.80) & 85 & (17.67) & 66 & (13.72) & 68 & (14.14) & 52 & (10.81) & 15 & (3.12) & 14 & (2.91) \\
\hline 1998 & 602 & (10.04) & 4 & $(0.66)$ & 5 & $(0.83)$ & 4 & (0.66) & 10 & (1.66) & 39 & (6.48) & 82 & (13.62) & 108 & (17.94) & 136 & (22.59) & 94 & (15.61) & 83 & (13.79) & 24 & (3.99) & 13 & $(2.16)$ \\
\hline 1999 & 477 & (7.95) & 3 & (0.63) & 5 & $(1.05)$ & 3 & $(0.63)$ & 7 & (1.47) & 17 & (3.56) & 63 & $(13.21)$ & 109 & $(22.85)$ & 129 & $(27.04)$ & 80 & (16.77) & 29 & (6.08) & 26 & (5.45) & 6 & $(1.26)$ \\
\hline 2000 & 437 & (7.28) & 4 & $(0.92)$ & 2 & $(0.46)$ & 2 & $(0.46)$ & 2 & $(0.46)$ & 11 & (2.52) & 44 & (10.07) & 101 & (23.11) & 154 & (35.24) & 85 & $(19.45)$ & 29 & (6.64) & 0 & $(0.00)$ & 3 & $(0.69)$ \\
\hline 2001 & 221 & (3.68) & 0 & $(0.00)$ & 1 & $(0.45)$ & 2 & $(0.90)$ & 1 & $(0.45)$ & 1 & $(0.45)$ & 8 & (3.62) & 41 & (18.55) & 139 & $(62.90)$ & 26 & (11.76) & 2 & $(0.90)$ & 0 & $(0.00)$ & 0 & $(0.00)$ \\
\hline 2002 & 399 & (6.65) & 8 & (2.01) & 5 & $(1.25)$ & 17 & $(4.26)$ & 20 & (5.01) & 19 & $(4.76)$ & 49 & (12.28) & 82 & (20.55) & 88 & (22.06) & 65 & (16.29) & 26 & (6.52) & 13 & (3.26) & 7 & (1.75) \\
\hline 2003 & 94 & (1.57) & 0 & $(0.00)$ & 1 & $(1.06)$ & 2 & (2.13) & 0 & $(0.00)$ & 1 & $(1.06)$ & 5 & (5.32) & 15 & (15.96) & 24 & (25.53) & 29 & (30.85) & 13 & (13.83) & 2 & (2.13) & 2 & (2.13) \\
\hline 2004 & 252 & (4.20) & 1 & $(0.40)$ & 0 & $(0.00)$ & 0 & $(0.00)$ & 0 & $(0.00)$ & 5 & (1.98) & 12 & $(4.76)$ & 56 & $(22.22)$ & 102 & $(40.48)$ & 48 & (19.05) & 18 & (7.14) & 5 & (1.98) & 5 & (1.98) \\
\hline 2005 & 148 & (2.47) & 4 & (2.70) & 1 & (0.68) & 3 & (2.03) & 0 & $(0.00)$ & 0 & $(0.00)$ & 1 & (0.68) & 24 & $(16.22)$ & 53 & (35.81) & 39 & (26.35) & 15 & (10.14) & 6 & $(4.05)$ & 2 & (1.35) \\
\hline 2006 & 117 & (1.95) & 0 & $(0.00)$ & 0 & $(0.00)$ & 2 & $(1.71)$ & 0 & $(0.00)$ & 4 & (3.42) & 2 & $(1.71)$ & 21 & (17.95) & 41 & (35.04) & 28 & (23.93) & 17 & (14.53) & 2 & (1.71) & 0 & $(0.00)$ \\
\hline 2007 & 78 & (1.30) & 1 & $(1.28)$ & 2 & (2.56) & 0 & $(0.00)$ & 1 & $(1.28)$ & 1 & $(1.28)$ & 3 & (3.85) & 17 & (21.79) & 38 & $(48.72)$ & 10 & $(12.82)$ & 3 & (3.85) & 0 & $(0.00)$ & 2 & (2.56) \\
\hline 2008 & 22 & $(0.37)$ & 1 & (4.55) & 0 & $(0.00)$ & 0 & $(0.00)$ & 1 & $(4.55)$ & 0 & $(0.00)$ & 1 & (4.55) & 1 & (4.55) & 6 & $(27.27)$ & 12 & (54.55) & 0 & $(0.00)$ & 0 & $(0.00)$ & 0 & $(0.00)$ \\
\hline 2009 & 36 & $(0.60)$ & 0 & $(0.00)$ & 0 & $(0.00)$ & 2 & (5.56) & 6 & (16.67) & 0 & $(0.00)$ & 1 & (2.78) & 1 & (2.78) & 16 & $(44.44)$ & 7 & (19.44) & 2 & (5.56) & 0 & $(0.00)$ & 1 & (2.78) \\
\hline 2010 & 26 & $(0.43)$ & 1 & (3.85) & 1 & (3.85) & 1 & (3.85) & 1 & (3.85) & 1 & (3.85) & 6 & (23.08) & 1 & (3.85) & 7 & (26.92) & 4 & (15.38) & 2 & (7.69) & 0 & $(0.00)$ & 1 & (3.85) \\
\hline Total & 5,999 & (100.00) & 82 & (1.37) & 46 & $(0.77)$ & 72 & $(1.20)$ & 91 & (1.52) & 260 & (4.33) & 666 & (11.10) & 1442 & (24.04) & 1660 & (27.67) & 978 & (16.30) & 440 & (7.33) & 170 & (2.83) & 92 & (1.53) \\
\hline
\end{tabular}


Table 3 Distribution of S. flexneri and S. sonnei serotypes among patients with shigellosis in Beijing between 1994 and 2010 (by age group)

\begin{tabular}{|c|c|c|c|c|c|c|c|c|c|c|c|c|c|c|c|c|}
\hline \multirow{3}{*}{$\begin{array}{c}\begin{array}{c}\text { Age } \\
\text { groups }\end{array} \\
0-2\end{array}$} & \multicolumn{16}{|c|}{ No. (\%) of isolates in serotypes } \\
\hline & \multirow{2}{*}{$\begin{array}{c}\text { Total } \\
358\end{array}$} & \multirow[b]{2}{*}{$(6.03)$} & \multicolumn{2}{|c|}{$\begin{array}{c}\text { S. flexneri } \\
\text { serotype 1a }\end{array}$} & \multicolumn{2}{|c|}{$\begin{array}{c}\text { S. flexneri } \\
\text { serotype } 1 \mathrm{~b}\end{array}$} & \multicolumn{2}{|c|}{$\begin{array}{c}\text { S. flexneri } \\
\text { serotype } 2 a\end{array}$} & \multicolumn{2}{|c|}{$\begin{array}{c}\text { S. flexneri } \\
\text { serotype } 2 b\end{array}$} & \multicolumn{2}{|c|}{$\begin{array}{c}\text { S. flexneri } \\
\text { serotype } 3 a\end{array}$} & \multicolumn{2}{|c|}{$\begin{array}{c}\text { S. flexneri } \\
\text { serotype 3b }\end{array}$} & \multicolumn{2}{|c|}{$\begin{array}{c}\text { S. flexneri } \\
\text { serotype } 4 a\end{array}$} \\
\hline & & & 8 & $(4.68)$ & 0 & $(0.00)$ & 198 & $(6.28)$ & 3 & $(2.17)$ & 2 & $(6.67)$ & 1 & (33.33) & 12 & (6.94) \\
\hline $2-5$ & 463 & (7.80) & 10 & (5.85) & 2 & (14.29) & 202 & $(6.41)$ & 9 & (6.52) & 1 & (3.33) & 1 & (33.33) & 19 & (10.98) \\
\hline $6-10$ & 667 & $(11.24)$ & 16 & (9.36) & 3 & (21.43) & 307 & (9.74) & 10 & (7.25) & 5 & (16.67) & 0 & (0.00) & 23 & (13.29) \\
\hline $11-15$ & 549 & $(9.25)$ & 12 & (7.02) & 0 & $(0.00)$ & 232 & (7.36) & 13 & $(9.42)$ & 0 & $(0.00)$ & 0 & $(0.00)$ & 20 & $(11.56)$ \\
\hline $16-20$ & 568 & $(9.57)$ & 17 & $(9.94)$ & 1 & (7.14) & 275 & $(8.72)$ & 16 & $(11.59)$ & 5 & $(16.67)$ & 0 & $(0.00)$ & 14 & (8.09) \\
\hline $21-25$ & 979 & (16.50) & 37 & (21.64) & 3 & $(21.43)$ & 601 & (18.84) & 31 & $(22.46)$ & 7 & (23.33) & 1 & (33.33) & 34 & (19.65) \\
\hline $26-30$ & 600 & $(10.11)$ & 19 & (11.11) & 0 & $(0.00)$ & 380 & (12.05) & 17 & $(12.32)$ & 3 & $(10.00)$ & 0 & $(0.00)$ & 11 & (6.36) \\
\hline $31-35$ & 420 & (7.08) & 18 & (10.53) & 1 & (7.14) & 281 & $(8.91)$ & 11 & (7.97) & 2 & $(6.67)$ & 0 & $(0.00)$ & 9 & (5.20) \\
\hline $36-40$ & 476 & (8.02) & 8 & (4.68) & 2 & (14.29) & 163 & (5.17) & 12 & (8.70) & 3 & $(10.00)$ & 0 & $(0.00)$ & 5 & (2.89) \\
\hline $41-45$ & 195 & (3.29) & 7 & (4.09) & 0 & $(0.00)$ & 130 & (4.12) & 3 & (2.17) & 0 & $(0.00)$ & 0 & $(0.00)$ & 5 & (2.89) \\
\hline $46-50$ & 126 & (2.12) & 4 & (2.34) & 1 & (7.14) & 68 & (2.16) & 3 & (2.17) & 0 & $(0.00)$ & 0 & $(0.00)$ & 9 & (5.20) \\
\hline $51-55$ & 116 & (1.95) & 3 & $(1.75)$ & 1 & (7.14) & 69 & (2.19) & 3 & (2.17) & 0 & $(0.00)$ & 0 & $(0.00)$ & 5 & (2.89) \\
\hline $56-60$ & 100 & (1.69) & 2 & $(1.17)$ & 0 & $(0.00)$ & 63 & (2.00) & 1 & $(0.72)$ & 0 & $(0.00)$ & 0 & $(0.00)$ & 2 & (1.16) \\
\hline $61-65$ & 123 & (2.07) & 3 & (1.75) & 0 & $(0.00)$ & 78 & $(2.47)$ & 1 & $(0.72)$ & 0 & $(0.00)$ & 0 & $(0.00)$ & 1 & $(0.58)$ \\
\hline $66-70$ & 89 & (1.50) & 1 & (0.58) & 0 & $(0.00)$ & 53 & (1.68) & 2 & (1.45) & 0 & $(0.00)$ & 0 & $(0.00)$ & 3 & (1.73) \\
\hline $71-75$ & 67 & (1.13) & 5 & (2.92) & 0 & $(0.00)$ & 43 & (1.36) & 3 & (2.17) & 2 & $(6.67)$ & 0 & $(0.00)$ & 0 & $(0.00)$ \\
\hline $76-80$ & 25 & $(0.42)$ & 1 & (0.58) & 0 & $(0.00)$ & 11 & (0.35) & 0 & $(0.00)$ & 0 & $(0.00)$ & 0 & $(0.00)$ & 1 & $(0.58)$ \\
\hline $81-85$ & 10 & $(0.17)$ & 0 & $(0.00)$ & 0 & $(0.00)$ & 5 & $(0.16)$ & 0 & $(0.00)$ & 0 & $(0.00)$ & 0 & $(0.00)$ & 0 & $(0.00)$ \\
\hline $86-90$ & 3 & $(0.05)$ & 0 & $(0.00)$ & 0 & $(0.00)$ & 1 & (0.03) & 0 & $(0.00)$ & 0 & $(0.00)$ & 0 & $(0.00)$ & 0 & $(0.00)$ \\
\hline Total & 5,934 & $(100.00)$ & 17 & (2.88) & 1 & $(0.24)$ & 3,160 & (53.25) & 13 & (2.33) & 3 & $(0.51)$ & 3 & $(0.05)$ & 17 & (2.92) \\
\hline
\end{tabular}


Table 3 Distribution of S. flexneri and S. sonnei serotypes among patients with shigellosis in Beijing between 1994 and 2010 (by age group) (Continued)

\begin{tabular}{|c|c|c|c|c|c|c|c|c|c|c|c|c|c|c|}
\hline \multirow{3}{*}{$\begin{array}{c}\begin{array}{c}\text { Age } \\
\text { groups }\end{array} \\
0-2\end{array}$} & \multicolumn{14}{|c|}{ No. (\%) of isolates in serotypes } \\
\hline & \multicolumn{2}{|c|}{$\begin{array}{c}\text { S. flexneri } \\
\text { serotype } 4 \mathrm{~b}\end{array}$} & \multicolumn{2}{|c|}{$\begin{array}{l}\text { S. flexneri } \\
\text { serotype 4c }\end{array}$} & \multicolumn{2}{|c|}{$\begin{array}{l}\text { S. flexneri } \\
\text { serotype } 5\end{array}$} & \multicolumn{2}{|c|}{$\begin{array}{l}\text { S. flexneri } \\
\text { serotype } 6\end{array}$} & \multicolumn{2}{|c|}{$\begin{array}{l}\text { S. flexneri } \\
\text { serotype } x\end{array}$} & \multicolumn{2}{|c|}{$\begin{array}{l}\text { S. flexneri } \\
\text { serotype y }\end{array}$} & \multicolumn{2}{|c|}{ S. sonnei } \\
\hline & 3 & $(6.38)$ & 0 & $(0.00)$ & 0 & $(0.00)$ & 5 & $(9.43)$ & 18 & $(10.11)$ & 9 & $(8.18)$ & 99 & $(6.04)$ \\
\hline $2-5$ & 0 & $(0.00)$ & 5 & (2.66) & 2 & $(6.67)$ & 4 & (7.55) & 13 & $(7.30)$ & 5 & $(4.55)$ & 190 & (11.59) \\
\hline $6-10$ & 3 & $(6.38)$ & 18 & $(9.57)$ & 5 & $(16.67)$ & 1 & $(1.89)$ & 18 & $(10.11)$ & 8 & $(7.27)$ & 250 & $(15.25)$ \\
\hline $11-15$ & 4 & $(8.51)$ & 15 & (7.98) & 2 & $(6.67)$ & 4 & (7.55) & 3 & $(1.69)$ & 2 & $(1.82)$ & 242 & $(14.77)$ \\
\hline $16-20$ & 15 & $(31.91)$ & 13 & $(6.91)$ & 2 & $(6.67)$ & 2 & $(3.77)$ & 11 & (6.18) & 19 & (17.27) & 178 & (10.86) \\
\hline $21-25$ & 5 & (10.64) & 57 & (30.32) & 1 & (3.33) & 9 & (16.98) & 44 & $(24.72)$ & 26 & (23.64) & 123 & (7.50) \\
\hline $26-30$ & 5 & (10.64) & 21 & (11.17) & 2 & $(6.67)$ & 10 & (18.87) & 21 & (11.80) & 17 & (15.45) & 94 & (5.74) \\
\hline $31-35$ & 3 & (6.38) & 12 & (6.38) & 1 & (3.33) & 7 & $(13.21)$ & 12 & $(6.74)$ & 6 & (5.45) & 57 & (3.48) \\
\hline $36-40$ & 4 & (8.51) & 10 & (5.32) & 1 & $(36.67)$ & 2 & (3.77) & 14 & (7.87) & 2 & $(1.82)$ & 240 & (14.64) \\
\hline $41-45$ & 0 & $(0.00)$ & 12 & (6.38) & 1 & (3.33) & 2 & (3.77) & 8 & (4.49) & 1 & $(0.91)$ & 26 & (1.59) \\
\hline $46-50$ & 1 & (2.13) & 9 & (4.79) & 1 & (3.33) & 1 & (1.89) & 1 & $(0.56)$ & 0 & $(0.00)$ & 28 & (1.71) \\
\hline $51-55$ & 2 & (4.26) & 7 & (3.72) & 0 & $(0.00)$ & 2 & (3.77) & 4 & (2.25) & 5 & (4.55) & 15 & $(0.92)$ \\
\hline $56-60$ & 1 & (2.13) & 2 & $(1.06)$ & 0 & $(0.00)$ & 1 & $(1.89)$ & 6 & (3.37) & 2 & $(1.82)$ & 20 & $(1.22)$ \\
\hline $61-65$ & 0 & $(0.00)$ & 3 & (1.60) & 1 & (3.33) & 1 & (1.89) & 0 & $(0.00)$ & 3 & (2.73) & 32 & (1.95) \\
\hline $66-70$ & 1 & (2.13) & 2 & (1.06) & 0 & $(0.00)$ & 1 & (1.89) & 2 & (1.12) & 3 & (2.73) & 21 & $(1.28)$ \\
\hline $71-75$ & 0 & $(0.00)$ & 0 & $(0.00)$ & 1 & (3.33) & 0 & $(0.00)$ & 1 & $(0.56)$ & 0 & $(0.00)$ & 12 & (0.73) \\
\hline $76-80$ & 0 & $(0.00)$ & 2 & (1.06) & 0 & $(0.00)$ & 1 & (1.89) & 1 & (0.56) & 2 & $(1.82)$ & 6 & $(0.37)$ \\
\hline $81-85$ & 0 & $(0.00)$ & 0 & $(0.00)$ & 0 & $(0.00)$ & 0 & $(0.00)$ & 1 & $(0.56)$ & 0 & $(0.00)$ & 4 & $(0.24)$ \\
\hline $86-90$ & 0 & $(0.00)$ & 0 & $(0.00)$ & 0 & $(0.00)$ & 0 & $(0.00)$ & 0 & $(0.00)$ & 0 & $(0.00)$ & 2 & $(0.12)$ \\
\hline Total & 47 & (0.79) & 188 & (3.17) & 3 & $(0.51)$ & 53 & (0.89) & 178 & (3.00) & 110 & (1.85) & 1,639 & (27.62) \\
\hline
\end{tabular}


S. sonnei is shown in Table 3. Adults aged between 21 and 25 years were the most commonly affected group ( $\mathrm{n}=978 ; 16.3 \%$ ), followed closely by children aged less than 6 years $(\mathrm{n}=821 ; 13.6 \%)$. S. flexneri 2 a and $S$. sonnei were recovered from patients in each age group, although most infections caused by $S$. sonnei were found in children ( $\mathrm{n}=1639 ; 48 \%$ ); children aged 0 to 5,6 to 10 , and 11 to 15 years accounted for $17.6 \%(n=289)$, $15.2 \%(n=250)$, and $14.8 \%(n=242)$ of the cases, respectively. S. flexneri 2 a occurred frequently in adults, especially those in the 21 to $25(\mathrm{n}=594 ; 18.8 \%)$ and 26 to $30(\mathrm{n}=380,12 \%)$ age groups, although a high percentage $(n=400 ; 12.7 \%)$ was found to affect children aged less than 6 years.

\section{Gender}

Information about patient gender was known for all of the patients infected with S. flexneri and S. sonnei; distribution was slightly biased toward male patients ( $\mathrm{n}=$ 3616; 60\%; Table 4). Unexpectedly, the ratio for some of the predominant serotypes of $S$. flexneri and S. sonnei differed in distribution between male and female patients. The most prevalent S. flexneri serotype (2a) was found more frequently in males $(63.5 \%, p<0.0001)$ than in females (36.5\%). This result suggests that in Beijing, males either have greater exposure or are more susceptible to this subserotype than females. Similarly, $S$. flexneri 5 affected more males than females (ratio of infected males to females, 28 [46\%]:5 [44\%]; $p<0.001$ ), although this serotype is not common in Beijing. By contrast, $S$. flexneri $2 \mathrm{~b}$ and 6 as well as $S$. sonnei were more often associated with women than with men $(p<0.003$, $p<0.04$, and $p<0.0001$, respectively).

\section{Discussion}

According to a multicenter shigellosis surveillance study that incorporated data from six Asian sites, including China, shigellosis incidence is approximately 100-fold higher in Asia than in industrialized countries [5]. Other reports in China have indicated that shigellosis is even more ubiquitous than previously thought $[18,19]$. These studies have generally selected underdeveloped areas as surveillance sites, with little current information available on the epidemiologic trends in large cities like Beijing.

To begin to address this knowledge gap, the present study tracked Shigella infections in the largest infectious hospital in Beijing over a period of 17 years (1994 to 2010), thus providing a picture of serotype distribution across seasons, ages, and gender. The changing trend of Shigella in Beijing over time was determined in this work. The results will be useful in providing information that can be used by policy makers to implement control strategies or to predict the efficacy of vaccines for the prevention of shigellosis.

This study also demonstrated the diversity of all four species in recent years; the overall numbers of $S$. flexneri and $S$. sonnei have decreased, whereas the numbers of $S$. dysenteriae and $S$. boydii have not. S. flexneri 2a, the dominant serotype since 1994, was found to have been overtaken by S. sonnei in 2000; however, S. flexneri remained the dominant species in Beijing until 2006.

Table 4 Distribution of the serotypes in Beijing from 1994 to 2010 (by gender)

\begin{tabular}{|c|c|c|c|c|c|c|}
\hline \multirow{2}{*}{$\begin{array}{c}\text { Shigella species } \\
\text { and serotypes }\end{array}$} & \multicolumn{4}{|c|}{ No. (\%) of isolates } & \multirow{2}{*}{ Total } & \multirow{2}{*}{$\begin{array}{c}\text { Chi-square test } \\
\text { p value }\end{array}$} \\
\hline & \multicolumn{2}{|c|}{ Female } & \multicolumn{2}{|c|}{ Male } & & \\
\hline S. flexneri serotype $1 \mathrm{a}$ & 59 & $(0.99)$ & 112 & $(1.89)$ & 171 & $p<0.03$ \\
\hline S. flexneri serotype $1 \mathrm{~b}$ & 6 & $(0.10)$ & 8 & $(0.13)$ & 14 & $p>0.1$ \\
\hline S. flexneri serotype $2 a$ & 1,152 & $(19.41)$ & 2008 & $(33.84)$ & 3,160 & $p<0.001$ \\
\hline S. flexneri serotype $2 b$ & 71 & $(1.20)$ & 67 & $(1.13)$ & 138 & $p<0.003$ \\
\hline S. flexneri serotype $3 a$ & 8 & $(0.13)$ & 22 & $(0.37)$ & 30 & $p>0.05$ \\
\hline S. flexneri serotype 3b & 2 & $(0.03)$ & 1 & $(0.02)$ & 3 & $p>0.1$ \\
\hline S. flexneri serotype $4 a$ & 71 & $(1.20)$ & 102 & $(1.72)$ & 173 & $p>0.05$ \\
\hline S. flexneri serotype 4b & 14 & $(0.24)$ & 33 & $(0.56)$ & 47 & $p>0.05$ \\
\hline S. flexneri serotype $4 \mathrm{c}$ & 75 & $(1.26)$ & 113 & $(1.90)$ & 188 & $p>0.05$ \\
\hline S. flexneri serotype 5 & 3 & $(0.05)$ & 27 & $(0.46)$ & 30 & $p<0.001$ \\
\hline S. flexneri serotype 6 & 29 & $(0.49)$ & 24 & $(0.40)$ & 53 & $p<0.04$ \\
\hline S. flexneri serotype $x$ & 68 & $(1.15)$ & 110 & $(1.85)$ & 178 & $p>0.05$ \\
\hline S. flexneri serotype y & 40 & $(0.67)$ & 70 & $(1.18)$ & 110 & $p>0.05$ \\
\hline S. sonnei & 719 & $(12.12)$ & 920 & $(15.50)$ & 1,639 & $p<0.0001$ \\
\hline Total & 2,317 & (39.00) & 3,617 & $(61.00)$ & 5,934 & \\
\hline
\end{tabular}


The prevailing Shigella spp. in developing countries is S. flexneri, whereas that in developed countries is S. sonnei [14]. The same transition of dominant Shigella species observed not only in Beijing but also in southern Vietnam [20] and in Dhaka [21], all of which have undergone rapid economic development in recent years; the phenomenon supporting the circulating species and serotypes may be considered as a marker of the socioeconomic climate in an individual setting [22]. This gross economic improvement may have positively affected the quality of drinking water and the overall behavioral change in personal hygiene. These changes in turn may have influenced host characteristics, including the reduction of overall malnutrition that leads to an improved immune response. The genetic mutation of non-virulent microbes into pathogenic ones [23] may have also played an important role in the emergence of $S$. sonnei.

In this study, the 2006 data were compared with that of another similar report based on a study in Henan Province, China, in 2006 [18]. Shigella infection in Beijing reached its peak in August 2006, whereas that in Henan Province reached its peak in July 2006. For the same period, the data from the China Meteorological Administration (http://cdc.cma.gov.cn/home.do) revealed that the highest temperature in Beijing was recorded in August, whereas that in Zhengzhou, Henan Province, was recorded in July. Rainfall for this year peaked in July for both provinces. The high temperatures appear to more related to the seasonality of the Shigella infection than to the amount of rainfall; the same has also been observed in southern Vietnam [20].

Interestingly, the top three most common $S$. flexneri serotypes from 1994 to 2010 were S. flexneri 2a, 4c, and $\mathrm{x}$; overall, S. flexneri 2a ranked first, but S. flexneri 4c was the most dominant S. flexneri in 2004 and 2005. The same result was reported in Henan Province in 2006, during which S. flexneri 4c was the most frequently isolated serotype [18]. The distribution of $S$. flexneri serotypes between Beijing and other areas in China was highly heterogeneous from site to site and year to year. The observation that S. flexneri 4c had become dominant in Beijing and Henan suggests the spread of S. flexneri 4c throughout China. This serotype was first isolated in the USSR in the 1980s [24]. Similar to Beijing, S. flexneri 4c became the most predominant serotype in Zhejiang Province [25]. The factors contributing to the rise and the fall of S. flexneri 4c in China have not yet been fully explored. This study also highlighted the observation that age influenced the serotype distribution within the Shigella spp. Skewed gender distribution toward males was found for S. sonnei infection, which affected more males than females in Beijing; the same was also observed in Henan Province, China [18].
In conclusion, the change across 17 years in dominant Shigella species from S. flexneri to S. sonnei was uncovered. The results would be important in the treatment and prevention of shigellosis or in the implementation of vaccine strategies. The results also highlights the need for continuous monitoring of Shigella in the future.

\section{Consent}

Written informed consent was obtained from the patient for the publication of this report and any accompanying images.

\section{Competing interests}

The authors declared that they have no competing interests.

\section{Authors' contributions}

YM, EC, CB, ZL, SC, JZ, HW, CZ performed the experiments and provided clinical samples and patient data, JZ analyzed the data, JDK and BZ wrote the first draft of the manuscript, FQ and ZW designed the study, supervised the experiments and contributed to the manuscript. All authors read and approved the final manuscript.

\section{Acknowledgments}

This study was funded by the Chinese Military Medical and Technology Twelfth Five-Year Science and Research Key Program Fund (BWS11C073). The authors are grateful to helpful suggestions of Åsa Sjöling from University of Gothenburg. The English writing was polished by Enpapers.

\section{Author details}

${ }^{1}$ Clinical Diagnostic Center, 302nd Hospital of the People's Liberation Army, Beijing 100039, PR China. Institute of Microbiology, Chinese Academy of Sciences, Beijing 100101, PR China. ${ }^{3}$ School of Basic Medical Sciences, Peking University, Beijing 100083, PR China.

Received: 6 June 2013 Accepted: 31 July 2013

Published: 7 August 2013

\section{References}

1. Kotloff KL, Winickoff JP, Ivanoff B, Clemens JD, Swerdlow DL, Sansonetti PJ, Adak GK, Levine MM: Global burden of Shigella infections: implications for vaccine development and implementation of control strategies. Bull World Health Organ 1999, 77:651-666.

2. Wang $X Y, D u L$, Von Seidlein $L, X u Z Y$, Zhang $Y L$, Hao $Z Y$, Han OP, Ma JC, Lee $H J$, Ali $M$, et al: Occurrence of shigellosis in the young and elderly in rural China: results of a 12-month population-based surveillance study. Am J Trop Med Hyg 2005, 73:416-422.

3. Allison GE, Verma NK: Serotype-converting bacteriophages and O-antigen modification in Shigella flexneri. Trends Microbiol 2000, 8:17-23.

4. Talukder KA, Islam Z, Islam MA, Dutta DK, Safa A, Ansaruzzaman M, Faruque AS, Shahed SN, Nair GB, Sack DA: Phenotypic and genotypic characterization of provisional serotype Shigella flexneri 1c and clonal relationships with $1 \mathrm{a}$ and $1 \mathrm{~b}$ strains isolated in Bangladesh. J Clin Microbiol 2003, 41:110-117.

5. von Seidlein L, Kim DR, Ali M, Lee H, Wang X, Thiem VD, Chaicumpa W, Agtini MD, Hossain A, et al: A multicentre study of Shigella diarrhoea in six Asian countries: disease burden, clinical manifestations, and microbiology. PLoS Med 2006, 3:e353.

6. Cohen D, Ashkenazi S, Green MS, Gdalevich M, Robin G, Slepon R, Yavzori M, Orr N, Block C, Ashkenazi l, et al: Double-blind vaccine-controlled randomised efficacy trial of an investigational Shigella sonnei conjugate vaccine in young adults. Lancet 1997, 349:155-159.

7. Coster TS, Hoge CW, VanDeVerg LL, Hartman AB, Oaks EV, Venkatesan MM, Cohen D, Robin G, Fontaine-Thompson A, Sansonetti PJ, Hale TL: Vaccination against shigellosis with attenuated Shigella flexneri 2a strain SC602. Infect Immun 1999, 67:3437-3443.

8. Kotloff KL, Noriega FR, Samandari T, Sztein MB, Losonsky GA, Nataro JP, Picking WD, Barry EM, Levine MM: Shigella flexneri 2a strain CVD 1207, 
with specific deletions in virG, sen, set, and guaBA, is highly attenuated in humans. Infect Immun 2000, 68:1034-1039.

9. Mel DM, Papo RG, Terzin AL, Vuksic L: Studies on vaccination against bacillary dysentery. 2 . Safety tests and reactogenicity studies on a live dysentery vaccine intended for use in field trials. Bull World Health Organ 1965, 32:637-645.

10. Ferreccio C, Prado V, Ojeda A, Cayyazo M, Abrego P, Guers L, Levine MM: Epidemiologic patterns of acute diarrhea and endemic Shigella infections in children in a poor periurban setting in Santiago, Chile. Am J Epidemiol 1991, 134:614-627.

11. Noriega FR, Liao FM, Maneval DR, Ren S, Formal SB, Levine MM: Strategy for cross-protection among Shigella flexneri serotypes. Infect Immun 1999, 67:782-788.

12. Jennison AV, Roberts F, Verma NK: Construction of a multivalent vaccine strain of Shigella flexneri and evaluation of serotype-specific immunity. FEMS Immunol Med Microbiol 2006, 46:444-451.

13. Jennison AV, Verma NK: Shigella flexneri infection: pathogenesis and vaccine development. FEMS Microbiol Rev 2004, 28:43-58.

14. Gupta A, Polyak CS, Bishop RD, Sobel J, Mintz ED: Laboratory-confirmed shigellosis in the United States, 1989-2002: epidemiologic trends and patterns. Clin Infect Dis 2004, 38:1372-1377.

15. Wang XY, Tao F, Xiao D, Lee H, Deen J, Gong J, Zhao Y, Zhou W, Li W, Shen B, et al: Trend and disease burden of bacillary dysentery in China (19912000). Bull World Health Organ 2006, 84:561-568.

16. Ye C, Lan R, Xia S, Zhang J, Sun Q, Zhang S, Jing H, Wang L, Li Z, Zhou Z, et al: Emergence of a new multidrug-resistant serotype $X$ variant in an epidemic clone of Shigella flexneri. J Clin Microbiol 2010, 48:419-426.

17. Zhao YL, Yao W, Li HX: Influencing Factors for Rural Drinking Water and Sanitation. J Environ Health 2009, 25:8-9.

18. Xia S, Xu B, Huang L, Zhao JY, Ran L, Zhang J, Chen H, Pulsrikarn C, Pornruangwong S, Aarestrup FM, Hendriksen RS: Prevalence and characterization of human Shigella infections in Henan Province, China, in 2006. J Clin Microbiol 2011, 49:232-242.

19. Qiu S, Wang Z, Chen C, Liu N, Jia L, Liu W, Wang L, Hao R, Zhang L, Wang $Y$, Song $\mathrm{H}$ : Emergence of a novel Shigella flexneri serotype $4 \mathrm{~s}$ strain that evolved from a serotype $\mathrm{X}$ variant in China. J Clin Microbiol 2011, 49:1148-1150

20. Vinh H, Nhu NT, Nga TV, Duy PT, Campbell Jl, Hoang NV, Boni MF, My PV, Parry C, Nga TT, et al: A changing picture of shigellosis in southern Vietnam: shifting species dominance, antimicrobial susceptibility and clinical presentation. BMC Infect Dis 2009, 9:204.

21. Das SK, Ahmed S, Ferdous F, Farzana FD, Chisti MJ, Leung DT, Malek MA Talukder KA, Bardhan PK, Salam MA, et al: Changing emergence of Shigella sero-groups in Bangladesh: observation from four different diarrheal disease hospitals. PloS one 2013, 8:e62029.

22. Chompook P, Samosornsuk S, von Seidlein L, Jitsanguansuk S, Sirima N, Sudjai S, Mangjit P, Kim DR, Wheeler JG, Todd J, et al: Estimating the burden of shigellosis in Thailand: 36-month population-based surveillance study. Bull World Health Organ 2005, 83:739-746.

23. Greenhill C: Infectious disease: Genome sequencing reveals how Shigella sonnei spread around the world. Nat Rev Gastroenterol Hepatol 2012, 9:487.

24. Pryamukhina NS, Khomenko NA: Suggestion to supplement Shigella flexneri classification scheme with the subserovar Shigella flexneri $4 \mathrm{c}$ phenotypic characteristics of strains. J Clin Microbiol 1988, 26:1147-1149.

25. Zhang W, Pan JC, Meng DM, Ye R, Wang HQ: [PFGE of Shigella flexneri $4 \mathrm{C}$ isolates from food-poisoning outbreaks and sporadic diarrhea patients]. Zhonghua yu fang yi xue za zhi [Chinese journal of preventive medicine] 2007, 41:50-53.

doi:10.1186/1757-4749-5-21

Cite this article as: Mao et al:: Changing trends and serotype distribution of Shigella species in Beijing from 1994 to 2010. Gut Pathogens 2013 5:21.

\section{Submit your next manuscript to BioMed Central and take full advantage of:}

- Convenient online submission

- Thorough peer review

- No space constraints or color figure charges

- Immediate publication on acceptance

- Inclusion in PubMed, CAS, Scopus and Google Scholar

- Research which is freely available for redistribution

Submit your manuscript at www.biomedcentral.com/submit
Ciomed Central 